\title{
APPLICATION OF ANALYTIC NETWORK PROCESS TO A FORECASTING OF ORAL HEALTH
}

\author{
Andreichicova O.N. \\ Moscow University of Printing Art \\ Radyshevskaya T.N. \\ Volgograd State Medical University \\ Russia \\ alexandrol@mail.ru
}

Keywords: ANP, caries, dentistry, forecasting, oral health, periodontium.

Summary: The paper describes the application of Analytic Network Process for modeling the influence of various factors on an oral health status. The model based on the experts' knowledge enables to reveal the factors, which have the profound effect on a health status of organism's systems, being examined in conditions of interdependence. The outcomes obtained are in conformity with observation data. Described procedure can be used for research and forecasting of a health status of the human organism in general.

\section{Introduction}

Analytic Network Process developed by Thomas L. Saaty (Saaty T.L., 2001) can be successfully applied to forecasting a functional state of various forms of human physiological activity using the knowledge accumulated by skilled doctors and physiologists. In this paper we consider the problem of modeling of oral health, taking account of a number of external and internal factors.

The forecasting of the onset and development of dental diseases is an urgent problem in modern dentistry, as the incidence of dental caries and periodontium pathologies is very high among the human population and their clinical course is progressive. Usually a disease forecasting is based on clinical presentations of an action of various adverse factors on hard tooth tissues and on periodontium. As is known, caries and inflammatory diseases of periodontium are caused by both local and general risk factors (Leont'ev V.K., Shestakov V.T. and Voronin V.F., 2003; Rybakov A.I. and Chelidze L.N., 1990).

The well known general risk factors are: content of fluorine in water; a daily diet; stresses; somatic diseases, which give rise to abnormalities of endocrine regulation, immunity and metabolism.

In this research we take into consideration the following local factors: the presence of carbohydrate food debris; the presence of microorganisms in the dental deposit; the abnormal composition and properties of the oral fluid. Besides, the resistance of hard tooth tissues (full value of structure, chemical composition, genetic code) is important for the onset of caries.

Analytic Network Process (ANP) proposes a constructing of a qualitative model describing an influence of external factors upon the system under study, and also interdependence of the system's elements. Such a model can be built with use of knowledge of high-skilled experts; and it represents the linguistic description of basic components, elements and factors, as well as a description of a meaning and directions of influence flows in the system researched. The influence of different factors upon the system's elements (or a mutual influence of internal elements) can be of ambiguous semantics, therefore at initial stage of research, one should precisely formulate a main goal, in terms of which the basic categories of the task will be determined: criteria, components or clusters, elements, judgments. 


\section{Constructing a network model}

The main goal of this research is to find out the factors, which make the greatest impact into oral health. The results of scientific research, published scientific works (Rybakov A.I. and Chelidze L.N., 1990; Eriksen H.M. and Bjertness E., 1991; Hollister M.C. and Weintraub J.A., 1993; Leont'ev V.K., Shestakov V.T. and Voronin V.F., 2003; Andreichicova O.N. and Radyshevskaya T.N., 2003), as well as the experience of applied medicine were used to single out 15 major factors influencing an oral health. These factors were grouped into the following 7 clusters.

1) Life-support conditions. This term reflects external influence of environmental factors upon a human organism, in particular, upon oral health. The most important factors among them are represented by the following elements of this cluster:

1.1. Nutrition compound (foodstuff composition; amount of consumed carbohydrates; softness or hardness of food);

1.2. Ecology of life (chemical composition of air and water, including content of fluorine in water; geoclimatic conditions of existence);

1.3. Social conditions (sanitary conditions depending on social environment and personal income).

2) Oral hygiene. Main external factors determining oral hygiene are:

2.1. Skills of proper tooth brushing;

2.2. Regularity of dental inspections;

2.3. Quality of hygienic means used.

Training patients to brush teeth properly and an individual selection of hygienic means for oral cavity decrease the risk of dental diseases.

3) Status of dental health . This cluster characterizes the current state of oral cavity conditioned by all external and internal influences, as well as by case history of a patient. There are two main elements here:

3.1. State of teeth;

3.2. State of periodontium.

State of teeth (presence of carious cavities, poor-quality tooth stoppings and dental deposits) in many respects determines the state of periodontium (onset and progress of gingivitis and periodontitis). Besides, the onset of periodontium diseases can be associated with dental anomalies and deformation, errors during prosthetic alignments, and also with dental traumas. Inflammatory diseases of periodontium influence both the general health of the human organism and the state of teeth. A formation of bone recesses at periodontitis finally results in loosening of teeth, while etiological microbe agent promotes caries of hard tooth tissues.

4) General health status. This cluster characterizes the influence of basic parameters of human health on the state of oral cavity. Among these parameters we consider the following elements:

4.1. Hormonal status;

4.2. Age;

4.3. Level of immune reactivity.

We suppose these parameters of the general health have important influence on oral health and their further detailed elaboration is not expedient.

5) A type of blood circulation is one of major internal factors influencing dental and periodontium health (Rybakov A.I. and Chelidze L.N., 1990). We shall examine integrated influence of this factor without detailing it by a set of elements and we shall presume that various types of blood circulation (eukinetic, hyperkinetic, hypokinetic) influence a state of other systems by different ways.

6) A type of vegetative regulation determines vascular tone, secretion of salivary glands, and also blood supply to teeth and periodontium. This cluster, as well as previous (a type of blood circulation), is considered at the generalized level, i.e. it is submitted by a single element. An influence of a vegetative regulation type (sympathotonic, normotonic and vagotonic) upon a general health of human organism as a whole and, in particular, upon an oral health is beyond question and it is described in a number of scien- 
tific works (Rybakov A.I. and Chelidze L.N., 1990; Eriksen H.M. and Bjertness E., 1991; Hollister M.C. and Weintraub J.A., 1993).

7) Salivation system. This cluster is detailed by a set of the following elements, whose influence upon oral health is well known (Borovskiy E.V. and Leont'ev B.K., 1991; Leont'ev V.K., Shestakov V.T. and Voronin V.F., 2003):

7.1. Saliva secretion rate;

7.2. Salivation character (qualitative and quantitative saliva composition).

Such factors as ethnic features, professional insalubrities, harmful habits etc. were not taken into account in the model because of their insignificant influence in comparison with the factors described above.

After the allocation of basic elements and clusters it is necessary to ascertain connections between them. The graph of clusters interaction is shown in a Figure 1 where the arrows specify influence directions. The arrow from one cluster to another testifies that all or some elements in the first cluster influence all or some elements in the second cluster. The loops of a feedback (internal cycles) correspond to mutual influence between the elements in one cluster. In addition to internal cycles, there can be external cycles formed by connections between different clusters. The examples of external cycles in a Figure 1 are the closed contours formed by the clusters 3 - 4 - 3, 4 - 6 - 4, 4 - 6 - 5 - 7 - 3 - 4, 4 - 7 - 3 - 4 etc. Let's consider in detail the meaning and direction of the connections between the clusters in a Figure 1.

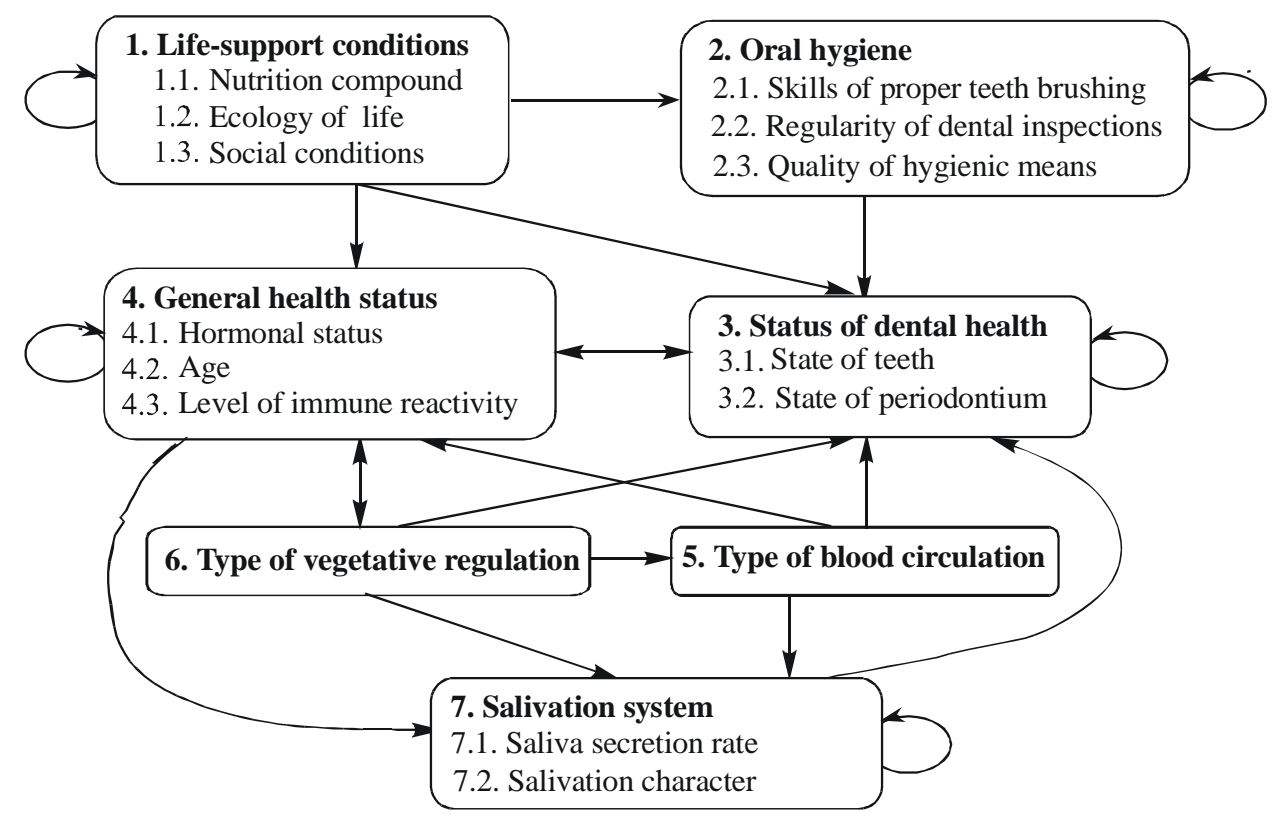

Figure 1 The influence graph describing a state of oral health

The first cluster's elements (Life-support conditions) noticeably influence a Status of dental health (cluster 3) and a General health status (cluster 4). Besides that, to a lesser degree, they influence each other, and some of them (Social conditions and Ecology of life) influence the elements of the second cluster (Oral hygiene). The most important influences of the first cluster's elements are the following ones: Social conditions $\rightarrow$ Nutrition compound, Social conditions $\rightarrow$ Quality of hygienic means, Nutrition compound $\rightarrow$ State of teeth, Ecology of life $\rightarrow$ Level of immune reactivity.

It is obviously, that the elements of second cluster (Oral hygiene) influence a State of dental health (cluster 3). A loop of a feedback at the second cluster is explained by an assumption, that a Regularity of dental inspections is under influence of other elements of this cluster. 
In the third cluster a State of teeth (presence of cavities, stoppings, teeth congestion) in many respects determines a State of periodontium, which, in turn, influences a State of teeth, i.e. the elements of this cluster influence each other. It's clear that the third and fourth clusters are interdependent, as insufficient mastication of food, presence of chronic infections' foci, and inflammations in oral cavity influence a General health status.

In turn, the fourth cluster's elements (Hormonal status, Age and Level of immune reactivity) influence a Status of dental health. Except direct affecting, a General health status marginally influences a Status of dental health through the seventh cluster (Salivation system). The type of vegetative regulation depends on elements of the fourth cluster, namely, a type can change with age, after sustained diseases and stresses.

The type of blood circulation influences a General health status, a Status of dental health (particularly a State of periodontium) and a Salivation system, as cardiovascular system provides a necessary level of vital activity and is the indicator of a human organism's adaptability.

The type of vegetative regulation influences a Type of blood circulation, a Salivation system, a Status of dental health and a General health status. The intensity of these influences may be different, but the fact of their existence is beyond question.

The elements of the seventh cluster (Salivation system) mutually influence each other and also a Status of dental health.

The following step of this research is to find out the relative intensity of influence for all elements of the system examined. Further, on the base of constructed model, limiting priorities of the elements are calculated. These priorities characterize a contribution of every element into main goal being formulated.

\section{Revealing of relative intensity of influence for clusters and their elements}

In ANP an intensity of influence is being estimated by the experts with use of pairwise comparison's procedure and the fundamental ratio scale (Saaty T.L., 2001). Pair comparison technique is the most universal method of measurements, as it can be applied at absence of any scales and standards, in particular at a measurement of intangible attributes. Comparing two objects with respect to a common attribute (criterion, property) the expert estimates a relative preference of one object over another, choosing a suitable estimation from the fundamental scale. The expert's comparisons of homogeneous objects with respect to each criterion are put in reciprocal matrix of pairwise comparisons.

For each pairwise comparisons matrix the right eigenvector is calculated, which is interpreted as the vector of relative priorities of the elements compared. The calculation of the normalized eigenvector for any pairwise comparisons matrix with dimension of $n$ generally enables to change $\left(n^{2}-n\right)$ judgments to $n$ estimations expressed by the numbers from a continuous interval $[0,1]$. Calculation of eigenvalue for any matrix of pair comparisons gives an opportunity to check out the expert's judgments in terms of consistency and transitivity (Saaty T.L., 2001). For our model (see Figure 1), it was necessary to fill 35 pairwise comparisons matrixes for elements and 7 matrixes for clusters. The latter were used for a calculation of the clusters' weight factors. The network model in a Figure 1 can be presented by the following matrix:

$B=$\begin{tabular}{r||lllllll||} 
Clusters & 1 & 2 & 3 & 4 & 5 & 6 & 7 \\
1 & 1 & 0 & 0 & 0 & 0 & 0 & 0 \\
2 & 1 & 1 & 0 & 0 & 0 & 0 & 0 \\
3 & 1 & 1 & 1 & 1 & 1 & 1 & 1 \\
4 & 1 & 0 & 1 & 1 & 1 & 1 & 0 \\
5 & 0 & 0 & 0 & 0 & 0 & 1 & 0 \\
6 & 0 & 0 & 0 & 1 & 0 & 0 & 0 \\
7 & 0 & 0 & 0 & 1 & 1 & 1 & 1
\end{tabular}


The unit elements in the matrix $B$ testify that the cluster specified at the top (identifier of a column) influence the cluster specified to the left (identifier of a row). For each column of this matrix the matrix of pairwise comparisons of corresponding clusters was filled. At filling of matrixes, experts answered a question: "What of two compared clusters (specified at rows) the given cluster (at the top of column) does influence more, and how much more?" or a question "What of the compared clusters does contribute into the given cluster more, and how much more?" At answering these questions, the experts used estimations from the fundamental ratio scale. For example, influence of the first cluster (Life-support conditions) upon other clusters is represented by the following matrix:

$$
\begin{array}{cc||cccc||c}
\boldsymbol{A}_{\mathbf{1}}= & \mathbf{1} & 1 & 2 & 3 & 4 & W \\
& 1 & \mathbf{1} & 1 & 1 / 3 & 1 / 5 & 0.100 \\
& 2 & 1 & \mathbf{1} & 1 / 3 & 1 / 5 & 0.100 \\
& 3 & 3 & 3 & \mathbf{1} & 1 / 2 & 0.283 \\
4 & 5 & 5 & 2 & \mathbf{1} & 0.517
\end{array}
$$

The elements of the first cluster influence each other and also the elements of the second, third and fourth clusters (see the first column in the matrix $B$ ). Let's consider the explanation of experts' estimations in the matrix $A_{1}$ containing clusters' pairwise comparisons obtained at answering the question "What of two clusters the first cluster (Life-support conditions) does influence more, and how much more?". We can see the first cluster's elements make significant impact into the fourth cluster and some less impact into the third cluster.

The second column of the matrix $B$ testifies that the cluster Oral hygiene influences itself and the third cluster, thus the pairwise comparisons matrix $A_{2}$ looks like:

$$
\boldsymbol{A}_{2}=\begin{array}{l||cc||c}
\mathbf{2} & \mathbf{2} & \mathbf{3} & W \\
\mathbf{2} & \mathbf{1} & \mathbf{1} & \mathbf{1} \\
\mathbf{3} & 5 & \mathbf{1} & 0.167 \\
& & & 0.833
\end{array}
$$

We can see that the factors of Oral hygiene influence a Status of dental health more, than each other.

Others 5 pairwise comparisons matrixes for clusters were similarly filled. Obtained priorities vectors were used for forming of the following matrix of clusters' weight factors:

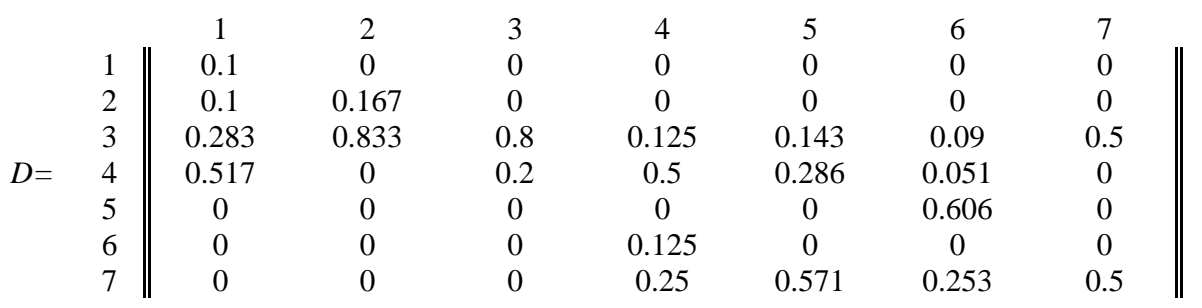

The next step is a filling of pairwise comparisons matrixes for elements of clusters and calculation of their priorities. Let's consider this procedure on the example of the first cluster, which contains the following elements: 1.1. - Nutrition compound; 1.2 - Ecology of life; 1.3 - Social conditions. These elements influence each other and also the elements of the second, third and fourth clusters. Some of them don't influence elements in other clusters. So, a Nutrition compound does not influence Ecology of life and Social conditions, however two the last factors influence other elements in the first cluster. We have the following pairwise comparisons matrixes for the first cluster's elements:

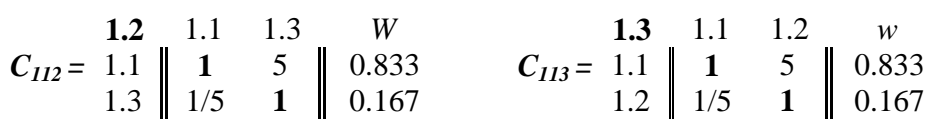

These matrixes demonstrate that Ecology of life influences Nutrition compound much more strongly, than Social conditions, and Social conditions influences Nutrition compound more strongly in comparison with Ecology of life. 
The second cluster's elements are influenced by all elements of the first cluster except Nutrition compound. Thus, Ecology of life (1.2) influences a Regularity of dental inspections (2.2) much more strongly, than other elements of the second cluster. The factor Social conditions (1.3) influences a Quality of hygienic means (2.3) in a greater extent than others and nearly does not influence a Skills of proper teeth brushing (2.1). These influences are represented by the following matrixes:

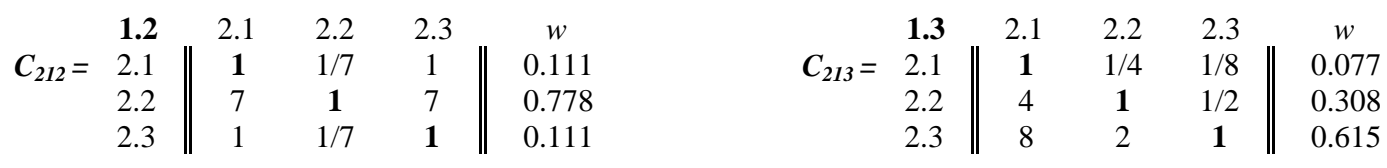

Nutrition compound (1.1) and Ecology of life (1.2) influence the elements of the third cluster (Status of dental health). The following matrixes contain estimations of intensity of their relative influence:

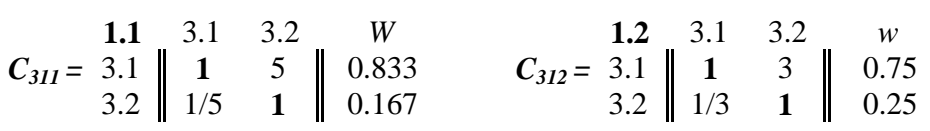

We can see from these matrixes that the elements of the first cluster influence a State of teeth more strongly, than a State of periodontium.

All elements of the first cluster influence the elements of the fourth cluster (General health status). These influences are brought in the following matrixes:

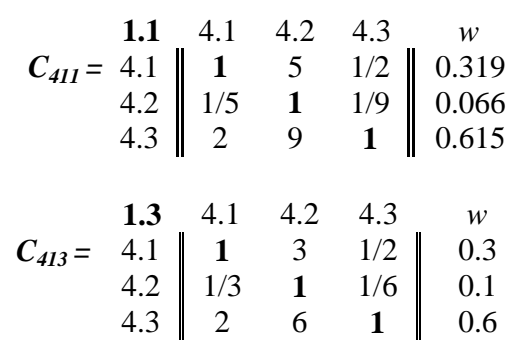

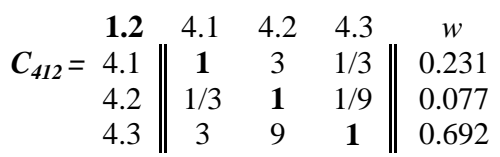

The matrixes above testify that Nutrition compound (1.1) exerts the most important influence on a Level of immune reactivity (4.3), lesser on a Hormonal status (4.1) and nearly does not influence Age (4.2). Ecology of life (1.2) and Social conditions (1.3) influence the elements of fourth cluster similarly.

The pairwise comparisons matrixes for estimation of influence of the elements from other clusters were similarly formed. The priorities vectors computed for these matrixes were put in the unweighted supermatrix for this network task, which is shown in Table 1. Expert judgments in all matrixes had a good consistency (index of a consistency did not exceed 0.02).

\section{Calculation of limiting priorities for the factors}

When elements in a complex system influence each other their priorities constantly change, therefore one can say the considered task becomes dynamic. We are interested with a long-term forecast of oral health and with a limit result of mutual influencing of the factors considered. The ANP enables to find out, whether there is a steady limit state for a network model with feedback and also to calculate limiting priorities for all elements and clusters.

If the sums of elements in every column of a supermatrix are equal to unity, then speak that the matrix is stochastic by columns. The consecutive raising of a stochastic matrix into integer powers results in a finite limit, which, generally speaking, may be non-unique. To make a supermatrix stochastic one can multiply its blocks corresponding to the clusters by the cluster's weight factors shown in a matrix $D$. The result is a weighed supermatrix, which is brought in Table 2. The raising of this matrix into high powers has resulted in a steady solution, which is a square matrix with identical columns, whose elements do not 
change at the further increasing of the exponent. These are the limiting priorities of the elements. We can see them in Table 3 and interpret as the factors' contributions into main goal.

Table 1 Unweighted supermatrix

\begin{tabular}{|c|c|c|c|c|c|c|c|c|c|c|c|c|c|c|c|}
\hline & \multicolumn{3}{|c|}{1} & \multicolumn{3}{|c|}{2} & \multicolumn{2}{|c|}{3} & \multicolumn{3}{|c|}{4} & \multirow{2}{*}{$\begin{array}{l}5 \\
5\end{array}$} & \multirow{2}{*}{$\begin{array}{l}6 \\
6\end{array}$} & \multicolumn{2}{|r|}{7} \\
\hline & 1.1 & 1.2 & 1.3 & 2.1 & $\begin{array}{ll}2.2 \\
\end{array}$ & 2.3 & 3.1 & \begin{tabular}{|l|l}
3.2 \\
\end{tabular} & 4.1 & 4.2 & 4.3 & & & 7.1 & 7.2 \\
\hline 1.1 & 0 & 0.833 & 0.833 & \multirow{3}{*}{\multicolumn{3}{|c|}{0}} & \multirow{3}{*}{\multicolumn{2}{|c|}{0}} & \multirow{3}{*}{\multicolumn{3}{|c|}{0}} & \multirow{3}{*}{0} & \multirow{3}{*}{0} & \multirow{3}{*}{\multicolumn{2}{|c|}{0}} \\
\hline 1.2 & 0 & 0 & 0.167 & & & & & & & & & & & & \\
\hline 1.3 & 0 & 0.167 & 0 & & & & & & & & & & & & \\
\hline 2.1 & 0 & 0.111 & 0.077 & 0 & 0.75 & 0.25 & \multirow{3}{*}{\multicolumn{2}{|c|}{0}} & \multirow{3}{*}{\multicolumn{3}{|c|}{0}} & \multirow{3}{*}{0} & \multirow{3}{*}{0} & \multirow{3}{*}{\multicolumn{2}{|c|}{0}} \\
\hline 2.2 & 0 & 0.778 & 0.308 & 0.875 & $\begin{array}{l}0 \\
0\end{array}$ & 0.75 & & & & & & & & & \\
\hline 2.3 & 0 & 0.111 & 0.615 & 0.125 & 0.25 & 0 & & & & & & & & & \\
\hline 3.1 & 0.833 & 0.75 & 0 & 0.75 & 0.833 & 0.833 & 0 & 1 & 0.167 & 0.5 & 0.25 & 0.2 & 0.25 & 0.75 & 0.333 \\
\hline 3.2 & 0.167 & 0.25 & 0 & 0.25 & 0.167 & 0.167 & 1 & 0 & 0.833 & 0.5 & 0.75 & 0.8 & 0.75 & 0.25 & 0.667 \\
\hline 4.1 & 0.319 & 0.231 & 0.3 & \multirow{3}{*}{\multicolumn{3}{|c|}{0}} & 0.222 & 0.236 & 0 & 0.667 & 0.833 & 0.236 & 0.25 & \multirow{3}{*}{\multicolumn{2}{|c|}{0}} \\
\hline 4.2 & 0.066 & 0.077 & 0.1 & & & & 0.111 & 0.082 & 0.25 & 0 & 0.167 & 0.082 & 0.069 & & \\
\hline 4.3 & 0.615 & 0.692 & 0.6 & & & & 0.667 & 0.682 & 0.75 & 0.333 & 0 & 0.682 & 0.681 & & \\
\hline 5 & \multicolumn{3}{|c|}{0} & \multicolumn{3}{|c|}{0} & \multicolumn{2}{|c|}{0} & \multicolumn{3}{|c|}{0} & 0 & 1 & \multicolumn{2}{|r|}{0} \\
\hline 6 & \multicolumn{3}{|c|}{0} & \multicolumn{3}{|c|}{0} & \multicolumn{2}{|c|}{0} & 1 & 1 & 1 & 0 & 0 & \multicolumn{2}{|r|}{0} \\
\hline 7.1 & \multirow{2}{*}{\multicolumn{3}{|c|}{0}} & \multirow{2}{*}{\multicolumn{3}{|c|}{0}} & \multirow{2}{*}{\multicolumn{2}{|c|}{0}} & 0.25 & 0.75 & 0.333 & 0.25 & 0.75 & 0 & 1 \\
\hline 7.2 & & & & & & & & & 0.75 & 0.25 & 0.667 & 0.75 & 0.25 & 1 & 0 \\
\hline
\end{tabular}

Table 2 Weighted supermatrix

\begin{tabular}{|c|c|c|c|c|c|c|c|c|c|c|c|c|c|c|c|}
\hline & \multicolumn{3}{|c|}{1} & \multicolumn{3}{|c|}{2} & \multicolumn{2}{|c|}{3} & \multicolumn{3}{|c|}{4} & 5 & 6 & \multicolumn{2}{|c|}{7} \\
\hline & 1.1 & 1.2 & $\begin{array}{l}1.3 \\
\end{array}$ & 2.1 & 2.2 & 2.3 & 3.1 & \begin{tabular}{|l|l}
3.2 \\
\end{tabular} & 4.1 & 4.2 & 4.3 & 5 & 6 & 7.1 & 7.2 \\
\hline 1.1 & 0 & 0.083 & 0.117 & \multirow{3}{*}{\multicolumn{3}{|c|}{0}} & \multirow{3}{*}{\multicolumn{2}{|c|}{0}} & \multirow{3}{*}{\multicolumn{3}{|c|}{0}} & \multirow{3}{*}{0} & \multirow{3}{*}{0} & \multirow{3}{*}{\multicolumn{2}{|c|}{0}} \\
\hline 1.2 & 0 & 0 & 0.023 & & & & & & & & & & & & \\
\hline 1.3 & 0 & 0.017 & 0 & & & & & & & & & & & & \\
\hline 2.1 & 0 & 0.011 & 0.011 & 0 & 0.125 & 0.042 & \multirow{3}{*}{\multicolumn{2}{|c|}{0}} & \multirow{3}{*}{\multicolumn{3}{|c|}{0}} & \multirow{3}{*}{0} & \multirow{3}{*}{0} & \multirow{3}{*}{\multicolumn{2}{|c|}{0}} \\
\hline 2.2 & 0 & 0.078 & 0.043 & 0.146 & 0 & 0.125 & & & & & & & & & \\
\hline 2.3 & 0 & 0.011 & 0.085 & 0.021 & 0.042 & 0 & & & & & & & & & \\
\hline 3.1 & 0.295 & 0.212 & 0 & 0.625 & 0.694 & 0.694 & 0 & 0.8 & 0.021 & 0.063 & 0.032 & 0.029 & 0.083 & 0.375 & 0.167 \\
\hline 3.2 & 0.039 & 0.071 & 0 & 0.208 & 0.139 & 0.139 & 0.8 & 0 & 0.104 & 0.063 & 0.094 & 0.115 & 0.067 & 0.125 & 0.333 \\
\hline 4.1 & 0.206 & 0.119 & 0.216 & \multirow{3}{*}{\multicolumn{3}{|c|}{0}} & 0.044 & 0.047 & & 0.333 & 0.416 & 0.067 & 0.013 & \multirow{3}{*}{\multicolumn{2}{|c|}{0}} \\
\hline 4.2 & 0.043 & 0.04 & 0.073 & & & & 0.023 & 0.017 & 0.125 & 0 & 0.083 & 0.023 & 0.004 & & \\
\hline 4.3 & 0.397 & 0.358 & 0.432 & & & & 0.133 & 0.136 & 0.375 & $\begin{array}{l}0.167 \\
\end{array}$ & 0 & 0.195 & 0.035 & & \\
\hline 5 & \multicolumn{3}{|c|}{0} & \multicolumn{3}{|c|}{0} & & 0 & \multicolumn{3}{|c|}{0} & 0 & 0.606 & \multicolumn{2}{|c|}{0} \\
\hline 6 & \multicolumn{3}{|c|}{0} & \multicolumn{3}{|c|}{0} & & 0 & $0.125 !$ & 0.125 & 0.125 & 0 & 0 & \multicolumn{2}{|c|}{0} \\
\hline 7.1 & \multirow{2}{*}{\multicolumn{3}{|c|}{0}} & \multirow{2}{*}{\multicolumn{3}{|c|}{0}} & \multirow{2}{*}{\multicolumn{2}{|c|}{0}} & $0.063:$ & 0.187 & 0.083 & 0.143 & 0.19 & 0 & 0.5 \\
\hline 7.2 & & & & & & & & & 0.187 & 0.063 & 0.167 & 0.428 & 0.063 & 0.5 & 0 \\
\hline
\end{tabular}

Table 3 Limiting priorities of the elements and clusters

\begin{tabular}{|c|c|c|c|c|c|c|c|c|c|c|c|c|c|}
\hline \multicolumn{3}{|c|}{1} & \multicolumn{3}{|c|}{2} & \multicolumn{2}{|c|}{3} & \multicolumn{3}{|c|}{4} & 5 & 6 & 7 \\
\hline \multicolumn{3}{|c|}{0} & \multicolumn{3}{|c|}{0} & \multicolumn{2}{|c|}{0.56} & \multicolumn{3}{|c|}{0.237} & 0.018 & 0.03 & 0.155 \\
\hline 1.1 & 1.2 & 1.3 & 2.1 & 2.2 & 2.3 & 3.1 & 3.2 & 4.1 & 4.2 & 4.3 & 5 & 6 & $\begin{array}{ll}7.1 & 7.2\end{array}$ \\
\hline 0 & 0 & 0 & 0 & 0 & 0 & 0.276 & 0.284 & 0.087 & 0.032 & 0.118 & 0.018 & 0.03 & 0.071 \\
\hline
\end{tabular}




\section{Results discussion}

The outcomes shown in Tables 2, 3 and in Figures 2, 3 suggest to conclude the following assertions about mutual influencing of external and internal factors:

- Life-support conditions have the most important influence on a State of teeth, a Level of immune reactivity and a Hormonal status.

- Oral hygiene strongly influences a State of teeth and less a State of periodontium.

- The elements of Dental health status noticeably affect each other. To a lesser degree they influence a Level of immune reactivity.

- Hormonal status has the most appreciable influence on a Level of immune reactivity, Salivation character and a State of periodontium.

- Age noticeably influences a Hormonal status, Saliva secretion rate, Level of immune reactivity and Vegetative regulation type.

- A Level of immune reactivity appreciably affects a Hormonal status and Salivation character.

- A Blood circulation type strongly influences Salivation character and less influences a Level of immune reactivity and a State of periodontium.

- A Vegetative regulation type governs a Blood circulation type. Besides, it noticeably influence Saliva secretion rate.

- The elements of Salivation system noticeably affect each other. They also have some influence on a Dental health status such that a Saliva secretion rate affects a State of teeth, and Salivation character - a State of periodontium.

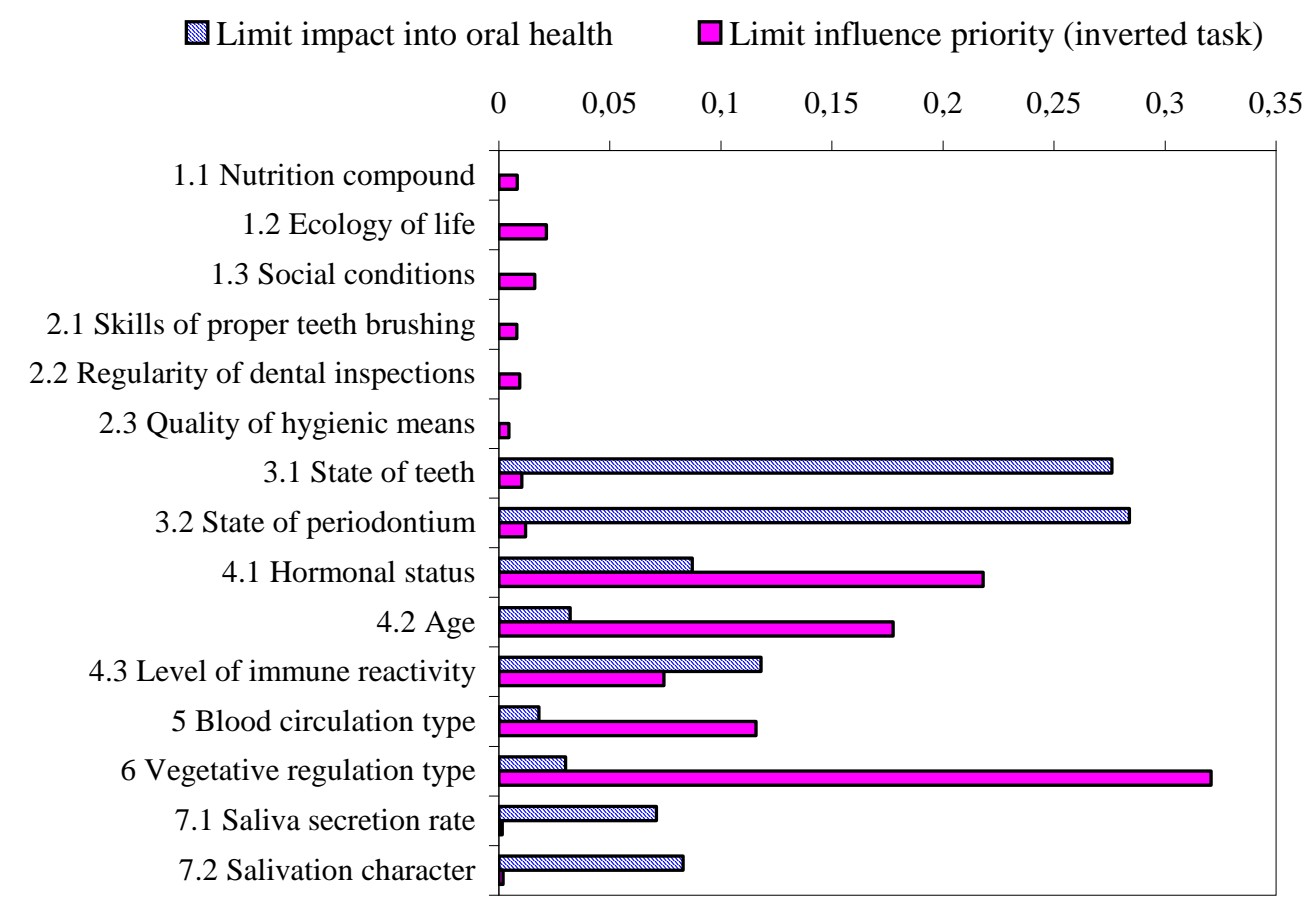

Figure 2 Limiting outcomes for the network model

The priorities shown in Tables 1, 2 can be considered as expert estimations of an influence intensity for the set of the examined factors at the present moment. Limiting priorities (see Table 3) can be interpreted as predictable values of the examined factors' contribution into main goal, in our case into oral health. The factors having high values of a limiting priority in a greater extent determine the purpose, as in the limit accumulate into itself the influence of other factors. In other words, data from Table 3 (and stripy columns in a Figures 2) correspond to the elements, which concentrate influence of all examined factors. It is easy to note, the elements of a Dental health status make the greatest impact into main goal, furthermore, the limiting priority of a State of periodontium is a little bit more, than priority of a State of teeth. The limiting priorities of the clusters are shown in a Figure 3 where the stripy columns correspond to the 
integrated contribution of every cluster into main goal. We can see that the contribution of the third cluster (Dental health status) exceeds $50 \%$. The third rank among all clusters' elements (see Figure 2) has a Level of immune reactivity, being the element of the fourth cluster (General health status), which takes the second place among all clusters (see Figure 3). Another element of this cluster - Hormonal status, has the fourth rank. The element Age is the least significant in the fourth cluster; it can be explained by negligible influence on it of other factors. The Salivation system has the third rank among clusters, and its elements are at the fifth and sixth places (see Figure 2). The Salivation character is slightly more significant than Saliva secretion rate. The fourth place among the clusters occupies a Type of vegetative regulation followed by a Type of blood circulation. The limiting priorities of the elements from the first and the second clusters equal to zero. This fact can be explained that external factors are not influenced by other elements, therefore there is no accumulation of influence in them. The node corresponding to the first cluster in a Figure 1 is a source; and the node, which corresponds to the second cluster, is a flow element with a loop of a feedback.

$\square$ Limit impact into main goal $\quad \square$ Limit influence priority (inverted task)

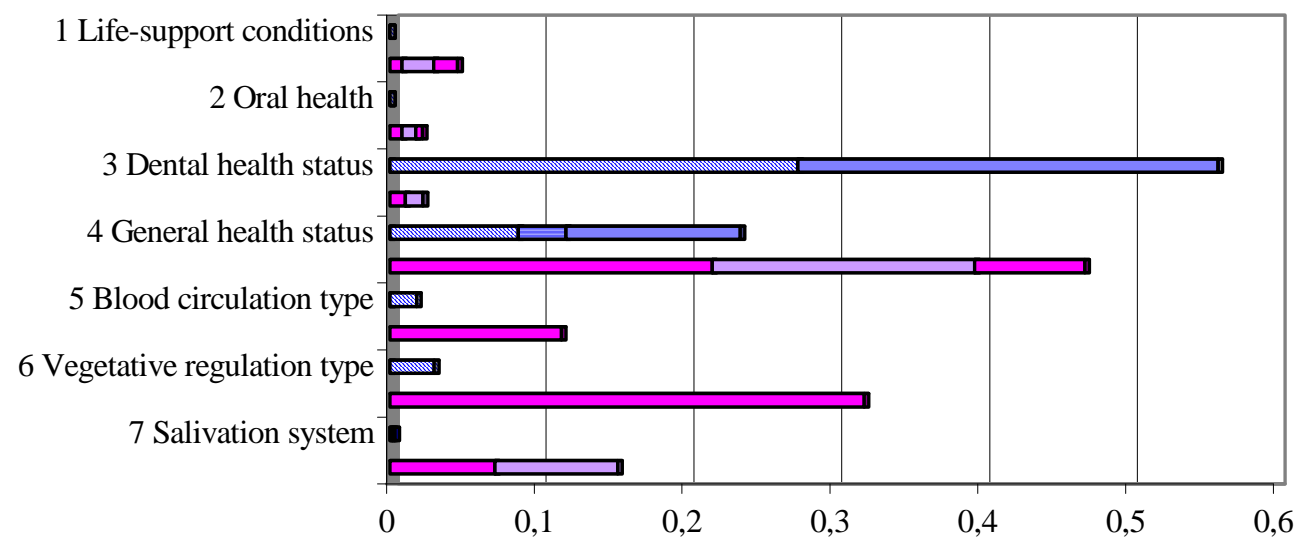

Figure 3 Limiting outcomes for the clusters

The first cluster is a powerful source influencing other clusters; only $10 \%$ of its influence makes up a feedback. The contribution of this cluster's elements into main goal comes through the third and fourth clusters indirectly (see above matrix $D$ ). The second cluster gets about $10 \%$ of the first cluster's influence, and only $16.7 \%$ of this value comes back through a feedback into second cluster. The contribution of the second cluster's elements into main goal is come through the third cluster (Dental health status).

In order to find out the elements, which have the greatest influence upon oral health, we inverted this task by transposing the matrix $B$. At that it was necessary to fill the set of new pairwise comparisons matrixes, asking the experts a question: "What of two analyzed factors (clusters, elements) influence the given factor (cluster or element) more?" For example, "What of the elements of a Dental health status (State of teeth or State of periodontium) does influence a Level of immune reactivity more?” The supermatrix of the inverted task is shown in Table 4. Its columns show the influences of the factors specified in rows (at the left) upon the element being the heading of a column. The raising of this matrix into high powers enables to compute the limiting priorities of influence of the examined factors upon all elements of the model. These priorities called as limiting influence priorities are represented in Table 5 and in a Figures 2, 3.

The outcomes of the inverted task reveal the most influential of the factors examined. The obvious leader among them is the Type of vegetative regulation with the limiting priority 0.3207 , the second is a Hormonal status (0.218), and the third is Age (0.1776). The distribution of clusters' integrated priorities is shown in a Figure 3 where we can see that the greatest influence upon oral health has a General health status (cluster 4) followed by the Type of vegetative regulation and the Type of blood circulation behind it. 
The influence of external factors (cluster 1 and 2) is less significant that is coordinated with up-to-date concepts in dentistry science (Eriksen H.M. and Bjertness E., 1991; Leont'ev V.K., Shestakov V.T. and Voronin V.F., 2003).

Table 4 The weighed supermatrix of the inverted task

\begin{tabular}{|c|c|c|c|c|c|c|c|c|c|c|c|c|c|c|}
\hline & \multicolumn{3}{|c|}{1} & \multicolumn{3}{|c|}{2} & \multicolumn{2}{|c|}{3} & \multicolumn{3}{|c|}{4} & 5 & 6 & 7 \\
\hline & 1.1 & 1.2 & 1.3 & 2.1 & 2.2 & 2.3 & 3.1 & 3.2 & 4.1 & 4.2 & 4.3 & 5 & 6 & $\begin{array}{l:l}7.1 & 7.2\end{array}$ \\
\hline 1.1 & 0 & 0 & 0 & 0 & 0.071 & 0.071 & 0.039 & 0.039 & 0.019 & 0 & 0.027 & \multirow{3}{*}{0} & \multirow{3}{*}{0} & \multirow{3}{*}{0} \\
\hline 1.2 & 0.2 & 0 & 0 & 0 & 0.214 & 0.071 & 0.013 & 0.013 & $0.056 ?$ & 0 & 0.054 & & & \\
\hline 1.3 & 0.8 & 0 & 0 & 0 & 0.214 & 0.357 & 0.006 & 0.006 & 0.019 & 0 & 0.014 & & & \\
\hline 2.1 & \multirow{3}{*}{\multicolumn{3}{|c|}{0}} & 0 & 0.333 & 0.167 & 0.176 & 0.176 & \multirow{3}{*}{\multicolumn{3}{|c|}{0}} & \multirow{3}{*}{0} & \multirow{3}{*}{0} & \multirow{3}{*}{0} \\
\hline 2.2 & & & & 0.75 & 0 & 0.333 & 0.062 & 0.062 & & & & & & \\
\hline 2.3 & & & & 0.25 & 0.167 & 0 & 0.033 & 0.033 & & & & & & \\
\hline 3.1 & \multirow{2}{*}{\multicolumn{3}{|c|}{0}} & \multirow{2}{*}{\multicolumn{3}{|c|}{0}} & 0 & 0.272 & 0.026 & 0 & 0.013 & 0 & 0 & 0 \\
\hline 3.2 & & & & & & & 0.272 & 0 & 0.026 & 0 & 0.04 & & & \\
\hline 4.1 & \multirow{3}{*}{\multicolumn{3}{|c|}{0}} & \multirow{3}{*}{\multicolumn{3}{|c|}{0}} & 0.064 & 0.122 & 0 & 0 & 0.324 & \multirow{3}{*}{0} & 0.571 & $\begin{array}{l:l}0.097 & 0.097\end{array}$ \\
\hline 4.2 & & & & & & & 0.02 & 0.03 & 0.324 & 0 & 0.108 & & 0.286 & $0.016: 0.016$ \\
\hline 4.3 & & & & & & & 0.128 & 0.06 & $0.108:$ & 0 & 0 & & 0.143 & $\begin{array}{l:ll}0.048 & 0.048 \\
\end{array}$ \\
\hline 5 & \multicolumn{3}{|c|}{0} & \multicolumn{3}{|c|}{0} & 0.03 & 0.03 & \multicolumn{3}{|c|}{$\begin{array}{l:l:l}0.155 & 0.368 & 0.155 \\
0.155\end{array}$} & 0 & 0 & $\begin{array}{l}0.083 \\
0.083 \\
\end{array}$ \\
\hline 6 & \multicolumn{3}{|c|}{0} & \multicolumn{3}{|c|}{0} & 0.037 & 0.037 & \multicolumn{3}{|c|}{$\begin{array}{l:l:l}0.266 & 0.632 & 0.266 \\
\end{array}$} & 1 & 0 & $\begin{array}{l:l}0.582 & 0.583\end{array}$ \\
\hline 7.1 & \multirow{2}{*}{\multicolumn{3}{|c|}{0}} & \multirow{2}{*}{\multicolumn{3}{|c|}{0}} & 0.078 & 0.029 & \multirow{2}{*}{\multicolumn{3}{|c|}{0}} & \multirow{2}{*}{0} & \multirow{2}{*}{0} & $\begin{array}{l:l}0 & 0.174\end{array}$ \\
\hline 7.2 & & & & & & & 0.039 & 0.087 & & & & & & $0.174 !$ \\
\hline
\end{tabular}

Table 5 Limiting priorities for the inverted task

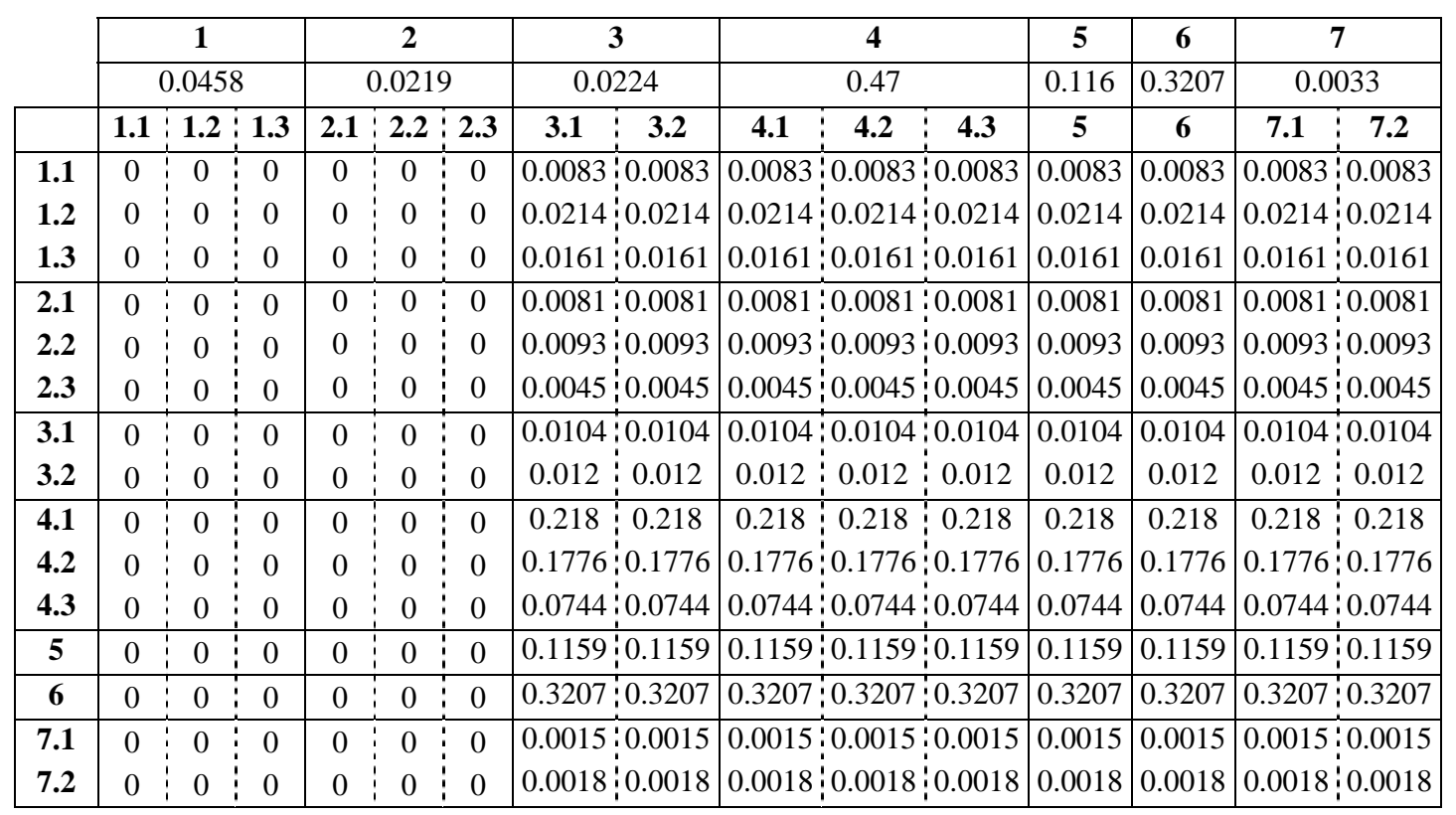

The Table 5 demonstrates that external factors collected in the first and second clusters are not influenced by internal ones. At the same time the limiting priorities of influence of each factor upon all other elements become the same. The general physiological factors (clusters $4,5,6$ ) have the most important influence upon oral health and others elements of the model. The influence of Life-support conditions (cluster 1) less, than total influence of above mentioned factors approximately in 10 times. The second cluster (Oral hygiene) and the third cluster (Dental health status) have small and close limiting priorities of influence, and the seventh cluster (Salivation system) is nearly insignificant. 
The results obtained are based on the study of pathological processes in the oral cavity in connection with other organism systems and a human environment. They are well matched with intuitive concepts of the experts, have reasonable interpretation, and enable to make a detailed forecast substantiation. The intermediate results also are of interest, as enable to analyze an opportunity of interaction of elements considered. The application of ANP gives an opportunity to estimate a contribution of the examined external and internal factors into the oral health status and to predict their influence in view of feedback in open physiological system.

The conclusion that the health of oral cavity is governed by the elements of a Dental health status is trivial and validates the expert information used. New results are the relative priorities describing influence intensity and impact into oral health of such factors as immunity level, hormonal status, vegetative regulation type, blood circulation type, and parameters of salivation system. These results are of a special interest of dentists and physiologists. The influence of the elements of a General health status is very strong; it's an order greater than the influence of external conditions. Let's note that the small limiting priorities of influence of the external factors should not be interpreted as the evidence of their insignificance. The cyclic character of mutual influence between the functional systems in human organism promotes the increasing of limiting priorities of these factors. For example, the elements of the third cluster (Dental health status) influence parameters of a General health status (cluster 4), which, in turn, influence a Dental health status, i.e. the influence in the cycle is gradually increased up to some limiting value. The clusters 1 and 2 representing external conditions are not included in cycles, therefore their impact into a Dental health status and into a General health status is distributed in the system and appears indirectly. This implies that the conditions of life-support and oral hygienic factors become more important when physiological parameters of the human organism are abnormal. In other words, people with immunodeficiency or with abnormalities of blood circulation, hormonal status and vegetative regulation are influenced by ecology, diet and social conditions in greater degree. Therefore they should care for the oral cavity more attentively and take preventive restorative measures.

The application of ANP to a problem of oral health forecasting has a number of advantages, in particular the following:

- An opportunity to build a model on the base of the expert information with taking into consideration the intangible factors and qualitative parameters;

- Considerable time saving and more wide area of the application in comparison with statistical models based on the empirical data;

- An opportunity to check out different hypotheses about a structure and intensity of influences at the various sets of the factors.

\section{References}

Andreichicova O.N. and Radyshevskaya T.N. (2003) “Application of Analytic Network Process for a forecasting of basic systems' health in the human organism”, Information Technologies, 7, 45-53 (in Russian).

Borovskiy E.V. and Leont'ev B.K. (1991) Biology of an oral cavity, Moscow: Medicine, (in Russian).

Eriksen H.M. and Bjertness E. (1991), “Concepts of health and disease and caries prediction: a literature review”, Scand. J. Dent. Res., 99 (6), 476-483.

Hollister M.C. and Weintraub J.A. (1993), "The association of oral status with systemic health, quality of life and economic productivity”, J. Dent. Educ., 57 (12), 901-912.

Leont'ev V.K., Shestakov V.T. and Voronin V.F. (2003) Estimation of the basic directions of dentistry development, Moscow: Medical book (in Russian).

Rybakov A.I. and Chelidze L.N. (1990), Anatomico-physiological features of an oral cavity and their significance in a pathology, Tbilisi: Metsnierba (in Russian).

Saaty T.L. (2001), Decision making with dependence and feedback. The analytic network process, Pittsburgh: RWS Publications. 